\title{
ISBER 2020 Awards
}

\author{
David Lewandowski ${ }^{1,2}$
}

$\mathrm{T}$ he International Society for Biological and Environmental Repositories (ISBER) award for outstanding achievement in biobanking, sponsored by Worthington Industries, is designed to recognize individuals who have made outstanding contributions to the field of biobanking. The award can be given for a single outstanding achievement or for a lifetime body of outstanding work in the field. ISBER is proud to announce the 2020 award winner of the ISBER outstanding achievement in biobanking award to Dr. Helen M. Moore.

Helen has a demonstrated history of making significant contributions to science and biobanking. Earlier in her career, she was part of the team at Celera Genomics, contributing to the first sequencing of the fruit fly, mouse, and human genomes. Furthering this effort, she led the development of web-based tools for scientists to access and use the data. After joining the biorepositories and biospecimen research team at the National Cancer Institute (NCI), she applied this knowledge and understanding to biospecimen science.

Helen led the development of numerous activities including the NCI's Biospecimen Research Network (BRN) program and the BRN symposium that brought needed recognition to biospecimen science as an understudied scientific discipline. Building on these efforts, she worked with colleagues at the NIH to develop and implement the Genotype-Tissue Expression (GTEx) project, partnering with organ procurement organizations and families to donate postmortem biospecimens that are the basis for an enormous genomic sequence resource being used all over the world.

Looking forward, Helen is excited to be leading a new program called the Cancer Moonshot Biobank. This initiative will establish a longitudinal biospecimen collection program focused on providing biospecimens for research in cancer treatment resistance. Helen is a proponent of increasing the transparency of our research world for patients and the general public, and incorporating engagement and ethical, legal, and social issues studies into biobanking. As we know in ISBER, biobanking in support of great science is a team effort and Helen has been fortunate to work with great teams that are trying to accomplish something significant to make the world a better place.
The ISBER Founder's Award, sponsored by Chart MVE Biomedical, recognizes individuals who have provided outstanding leadership to the founding, support, and incorporation of ISBER as an international biobanking society. ISBER is proud to announce Frank Simione as the 2020 recipient of the ISBER Founder's Award.

Frank Simione was an advocate early on, supporting the first meetings in many ways. In his career, Frank spent $>35$ years with the ATCC (American Type Culture Collection) of which he was a member of the ATCC senior management team for over half of this time. He was ATCC Director of Operations (1988-1996), managed ATCC's Patent Depository, the largest of 37 International Depository Authorities (IDAs) for patent purposes (1995-2005), and ATCC's safe deposit service (1980-2005). He also managed ATCC's regulatory compliance program for the safe handling, storage, and transport of biological materials (1999-2005), ATCC's Environmental Health and Safety Program (19882004), and ATCC's Select Agent Program (1997-present), and has provided institutional oversight for ATCC activities involving animal welfare, human subjects research (IRB Chair from 1993 to 2008), and biosafety for genetically modified materials.

In addition to being a founding member of ISBER, Frank is a past president of the ISBER.

The ISBER distinguished leadership and service award is designed to honor ISBER members who demonstrate exceptional leadership in supporting the mission and goals of the society and/or significant long-standing contributions to the society. ISBER is proud to announce Kathi Shea as the 2020 recipient of the ISBER distinguished leadership and service award.

Kathi's colleagues recognize the immense value of the experience she has gained through three decades of building sample collections for government, academic, and pharmaceutical research initiatives. She has served as an ISBER board member in various roles including director, secretary-treasurer, and president for a combined total of 8 years. During her term as president, Kathi helped lead ISBER as it transitioned to a new management partner and established itself as an official independent nonprofit organization. She authored sections of the initial version of ISBER best practices and has continued to offer her expertise with contributions to subsequent versions

\footnotetext{
${ }^{1}$ Brooks Life Sciences, Chelmsford, Massachusetts.

${ }^{2}$ Immediate-Past President ISBER.
} 
and her time on numerous ISBER committees and working groups.

Beyond ISBER, Kathi participated in the development of the NCI best practices for biospecimen resources, served on the advisory working group that developed the College of American Pathologists Biorepository Accreditation Program (CAP BAP), served on the CAP BAP committee for 6 years, and is an active CAP inspector. She continues her involvement with ISBER and especially enjoys teaching workshops on various aspects of sample management for ISBER and SLAS.

ISBER's special service awards recognize individuals who have made exceptional contributions toward the goals of the society through the leadership of a special project or act on behalf of the organization. ISBER is proud to announce this year's awardees:

- Marianne Henderson, for her many years of leadership and dedication to ISBER and the global biobanking field. Ms. Henderson has served as the chair of the organization advisory committee for the past 6 years, which is charged with the development of the annual meeting scientific program. She continues to mentor future leaders and has paved the way for the inclusion of low-to middle-income countries (LMIC) to have access to valuable biobanking procedures and policies.

- Diane McGarvey, for her focus on the advancement of the technician level and biobanking 101 workshops. Ms. McGarvey has served as the vice chair of the education and training workshops for the past few years, ensuring that the topics of the educational workshops meet the ever-growing needs and challenges of the biobanking community. She has focused on including a specialized workshop that focuses on the needs of the technicians and entry-level biobankers to ensure they have the tools to continue in the biobanking field.

- Brent Schacter, for his leadership on the development of the qualification in biorepository science program. Dr. Schacter has been championing this initiative as part of ISBER's goal to have a program that has global recognition as indicative of an individual who has skills, knowledge, and proficiency to play a role in the biobanking enterprise. In time it will become the global standard for demonstrated quality in a biobanker.

- Dayong Gao, for his vision to encourage ISBER and the Society of Cryopreservation to collaborate on activities that contribute to the overall goals of the two societies. Dr. Gao was instrumental in driving the codevelopment of the liquid nitrogen-based cryogenic storage of specimens addendum to ISBER's 4th edition of best practices for biorepositories, which was released in 2019.

- Jason Chen, for his role as a cultural advisor during the ISBER 2019 annual meeting held in Shanghai-our first meeting in China. Mr. Chen was instrumental in working with the presidents, board, and the China Director at Large (DAL) and Regional Ambassadors (RAs) to ensure that information on the scientific program and timelines was relayed to the appropriate parties. He worked diligently with the head office to have his team at China National GeneBank (CNGB) create and maintain the China ISBER website, ensuring that information is delivered in a timely manner.

The ISBER travel award was established $>10$ years ago and has made it possible for 14 individuals from LMICs to attend the ISBER annual meeting. The travel award is targeted toward individuals interested in either establishing or already managing a biorepository in their particular country or region. ISBER hopes that awardees will gain useful information from the various symposiums and workshops, network with experienced leaders in the biobanking field, and subsequently bring back valuable information from the meeting to help inform their colleagues and home country.

The eligibility criteria for the ISBER travel award are as follows: (1) the applicant is an employee of a not-for-profit LMIC biorepository organization, (2) the applicant is not a previous travel award winner or recipient, and (3) the applicant has not attended a previous ISBER meeting. In addition to providing a $\mathrm{CV}$, travel award applicants must describe their biobank experience and explain how meeting attendance will benefit them and acquired knowledge will be shared. The ISBER education and training committee reviews the applications and awardees are provided with airfare, hotel, a daily stipend, and complimentary attendance to the meeting event gala and $5 \mathrm{~K}$ Fun Walk/Run.

ISBER is able to fund one applicant's attendance to the 2020 ISBER annual meeting in Anaheim, CA. There were 12 eligible applicants representing the countries of Rwanda, Ghana, Egypt, Kenya, Uganda, India, and Pakistan.

Dr. Bushra Allah Rakha, assistant professor at the Department of Wildlife Management, Pir Mehr Ali Shah, Arid Agriculture University in Rawalpindi, Pakistan, was chosen as the 2020 ISBER travel award recipient. Dr. Rakha has been working on the conservation of threatened species for $>13$ years and has pioneered research on the development of modern reproductive technologies for multiple bird species. Dr. Rakha hopes attendance at the ISBER annual meeting will enable her to develop cryopreservation protocols and address the impacts of biobanks on species retrieval. After the meeting, Dr. Rakha hopes to bring awareness of Pakistan's most neglected species and the importance of their conservation/welfare by the creation of semen banks. In addition, she will prepare a collaborative research project and submit to international funding agencies to facilitate development of this important topic that is very new for Pakistan. ISBER is very excited to aid Dr. Rakha in these endeavors!

Address correspondence to: David Lewandowski Brooks Life Sciences 15 Elizabeth Drive Chelmsford, MA 01824

E-mail: david.lewandowski@brooks.com 